\title{
Fission modelling with FIFRELIN ${ }^{\star}$
}

\author{
Olivier Litaize $^{\mathrm{a}}$, Olivier Serot, and Léonie Berge \\ CEA, DEN, DER, SPRC, F-13108 Saint Paul Lez Durance, France
}

Received: 27 February 2015 / Revised: 11 June 2015

Published online: 23 December 2015 - (C) Società Italiana di Fisica / Springer-Verlag 2015

Communicated by N. Alamanos

\begin{abstract}
The nuclear fission process gives rise to the formation of fission fragments and emission of particles $\left(n, \gamma, e^{-}\right)$. The particle emission from fragments can be prompt and delayed. We present here the methods used in the FIFRELIN code, which simulates the prompt component of the de-excitation process. The methods are based on phenomenological models associated with macroscopic and/or microscopic ingredients. Input data can be provided by experiment as well as by theory. The fission fragment deexcitation can be performed within Weisskopf (uncoupled neutron and gamma emission) or a HauserFeshbach (coupled neutron/gamma emission) statistical theory. We usually consider five free parameters that cannot be provided by theory or experiments in order to describe the initial distributions required by the code. In a first step this set of parameters is chosen to reproduce a very limited set of target observables. In a second step we can increase the statistics to predict all other fission observables such as prompt neutron, gamma and conversion electron spectra but also their distributions as a function of any kind of parameters such as, for instance, the neutron, gamma and electron number distributions, the average prompt neutron multiplicity as a function of fission fragment mass, charge or kinetic energy, and so on. Several results related to different fissioning systems are presented in this work. The goal in the next decade will be i) to replace some macroscopic ingredients or phenomenological models by microscopic calculations when available and reliable, ii) to be a support for experimentalists in the design of detection systems or in the prediction of necessary beam time or count rates with associated statistics when measuring fragments and emitted particle in coincidence iii) extend the model to be able to run a calculation when no experimental input data are available, iv) account for multiple chance fission and gamma emission before fission, v) account for the scission neutrons. Several efforts have already been made to replace macroscopic ingredients and phenomenology by microscopic ingredients provided in various nuclear parameter libraries such as electric dipole photon strength functions or HFB level densities. First results relative to theses aspects are presented in this work.
\end{abstract}

\section{Introduction}

The fission process gives rise to the formation of fission fragments $(\mathrm{FF})$ and emission of particles $\left(n, \gamma, e^{-}\right)$. Primary fission fragments created just after scission are accelerated by Coulomb repulsion and evaporate prompt neutrons, gammas and conversion electrons. Fission products that are created in this way are unstable due to neutron excess and decay through $\left(\beta^{-}\right)$or $\left(\beta^{-}, n\right)$. Up to recently, a Weisskopf statistical theory [1] was used to simulate the prompt neutron evaporation from Monte Carlo algorithms (and sometimes also gammas) [2-10]. The Weisskopf approximation can be replaced by a more accurate treatment of the de-excitation process based on the so-called Hauser-Feshbach statistical theory [11] which allows ac-

\footnotetext{
* Contribution to the Topical Issue "Perspectives on Nuclear Data for the Next Decade" edited by Nicolas Alamanos, Eric Bauge, Stéphane Hilaire.

${ }^{a}$ e-mail: olivier.litaize@cea.fr
}

counting for the spins and parities of fission fragment nuclear levels involved during the whole neutron/gamma cascade $[12,13]$. We present here, in sect. 2 , the model used in the FIFRELIN Monte Carlo code to simulate the characteristics of the fission fragments and the prompt component (before beta decay) of the de-excitation process. Following sections aim at presenting some comparisons with experimental results before concluding and dealing with the perspectives of the next decade.

\section{Model}

The hypotheses under the model described hereafter are the following:

- Fission is a binary process (ternary fission is neglected).

- Fission fragments are assumed to recover their ground state deformation during acceleration (so called "relaxation" phase).

- Neutron emission at scission and during acceleration is not considered. 


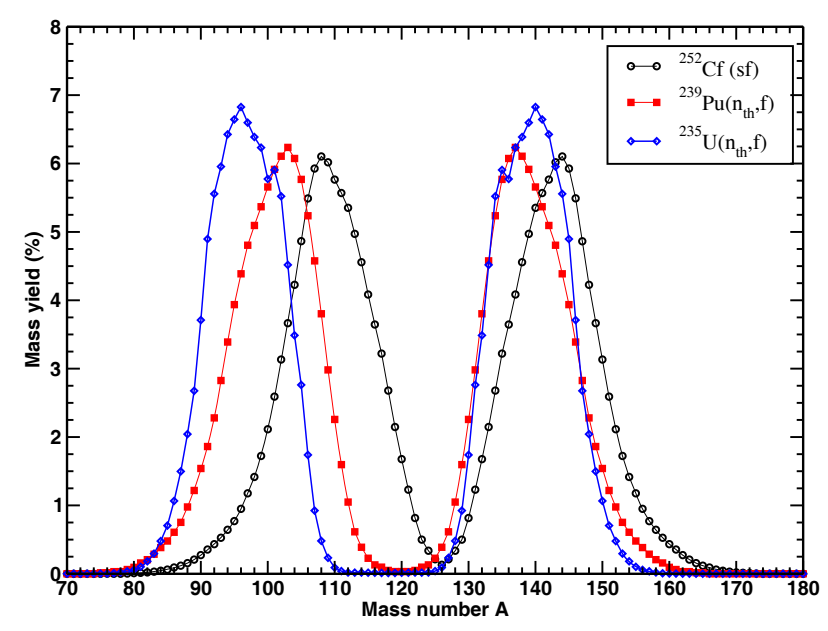

Fig. 1. Pre-neutron fission fragment mass yields (input data).

- Neutrons and gammas are emitted after this acceleration phase from fragments in their ground state deformation.

The first step of the model, e.g. the sampling of FF characteristics (mass $A$, nuclear charge $Z$, kinetic energy $K E$, excitation energy $E^{*}$, spin $J$ and parity $\pi$ ), has been previously described in [6]. The second step consists in decaying the completely characterized excited fragments. We briefly remind hereafter the principle.

\subsection{Mass charge and kinetic energy sampling}

In the actual release of the code, the pre-neutron mass and kinetic energy distributions are taken from experiments or calculated in the framework of the Multi-Modal Random Neck-Rupture model [14]. In that case, experimentalists furnish the fission mode weights by comparing data and calculations. We have reported in figs. 1, 2 and 3 the mass and kinetic energy distributions provided by experimentalists [15] for ${ }^{252} \mathrm{Cf}(s f),[16]$ for ${ }^{235} \mathrm{U}\left(n_{\mathrm{th}}, f\right)$ and $[17,18]$ for ${ }^{239} \mathrm{Pu}\left(n_{\mathrm{th}}, f\right)$ reactions. These results reported in the present work have been obtained with these experimental input data.

The nuclear charge is sampled from the Unchanged Charge Density (UCD) hypothesis modified by mass dependent polarization charge function (an oscillating structure has been observed in the polarization function as a function of mass $\Delta Z(A)$ for various fissioning systems [19-22]) and even-odd factors experimentally determined (when available).

\subsection{Spin and parity sampling}

The parity has simply a probability $1 / 2$ to be positive or negative. The spin is sampled from a kind of Rayleigh distribution accounting for the so-called spin cut-off parameter $\sigma^{2}$

$$
P(J)=\frac{2 J+1}{2 \sigma^{2}} \exp \left\{-\frac{(J+1 / 2)^{2}}{2 \sigma^{2}}\right\} .
$$

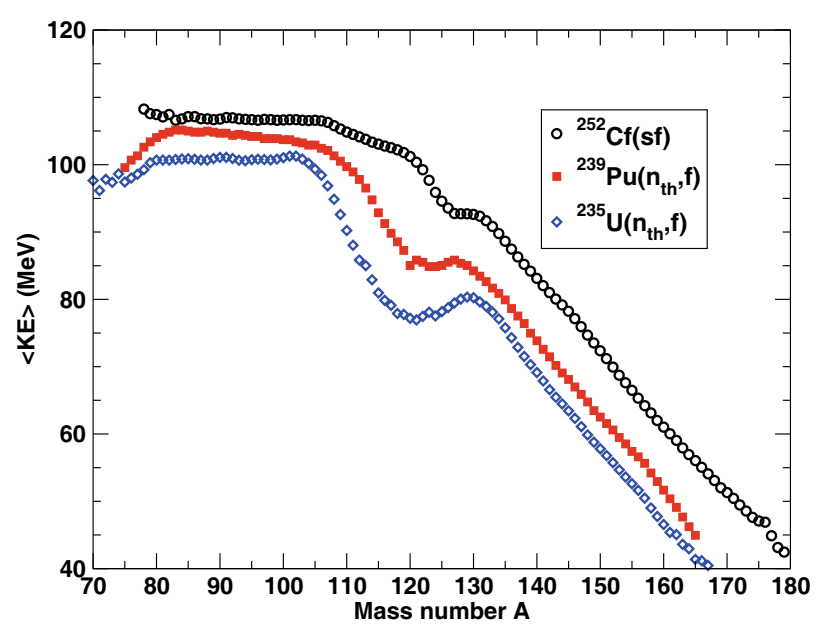

Fig. 2. Pre-neutron fission fragment average kinetic energy (input data).

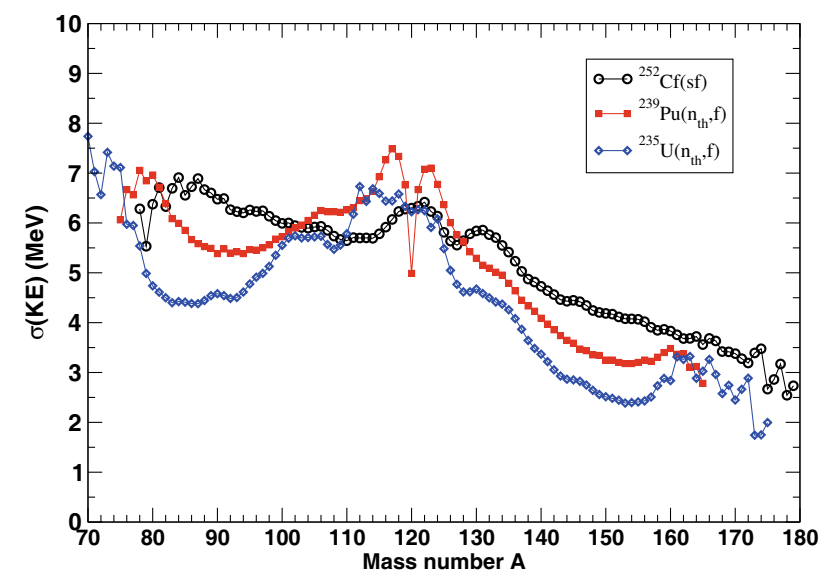

Fig. 3. Standard deviation of the pre-neutron fission fragment kinetic energy distributions (input data).

The spin cut-off parameter can be constant or excitation energy (temperature) dependent (see previous results reported in [23]). The temperature-dependent model involves also the moment of inertia of the fragment. In that case it is a fraction of a deformed rigid spheroid and the spin sampling is linked to the determination of the excitation energy sharing through an implicit equation. In this work, to select the primary fission fragment spin at the very beginning of the de-excitation process, two constant average spin cut-off parameters are used for the light fragment group $\left(\overline{\sigma_{L}^{2}}\right)$ and the heavy fragment group $\left(\overline{\sigma_{H}^{2}}\right)$.

\subsection{Excitation energy calculation}

The final quantity required to evaporate particles from an excited nucleus is the excitation energy which is not directly sampled because only the total excitation energy of the system is known at this step. We suppose that total excitation energy $T X E$ at scission is composed of intrinsic excitation energy $E^{*, \mathrm{sc}}$, deformation energy $E^{\text {def,sc }}$ (difference between potential energy corresponding to scission 


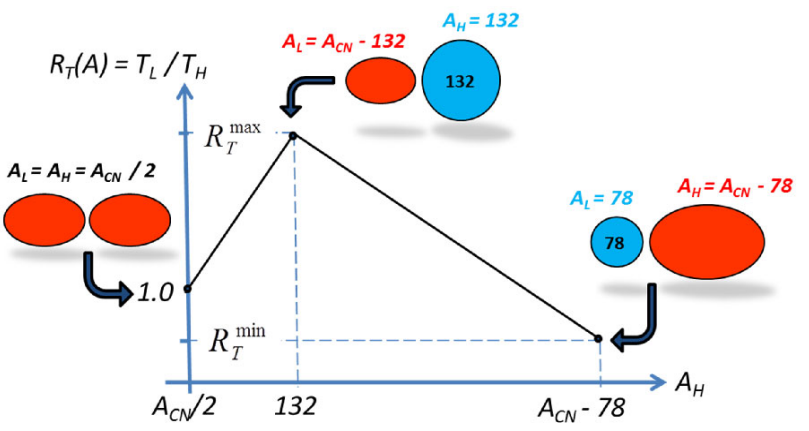

Fig. 4. Temperature ratio law $R_{T}(A)$ : three anchorage points are considered from macroscopic nucleus shapes at scission to determine the temperature ratio of two complementary fission fragments after full acceleration when they have recovered their ground state deformation (see text for details).

deformation and potential energy corresponding to ground state deformation) and collective excitation modes:

$$
T X E=E^{*, \mathrm{sc}}+E^{\mathrm{def}, \mathrm{sc}}+E^{\mathrm{coll}, \mathrm{sc}} .
$$

The collective part of the excitation energy is assumed to be rotational in a first approximation. After full acceleration during which relaxation of the deformation energy is assumed, the total excitation energy is converted into intrinsic excitation energy $E^{*}$ and collective rotational energy $E^{\text {rot }}$

$$
T X E=E_{L}^{*}+E_{H}^{*}+E_{L}^{\mathrm{rot}}+E_{H}^{\mathrm{rot}},
$$

where $E_{L}^{\text {rot }}, E_{H}^{\text {rot }}$ are the rotational energies of the light and heavy fragments and $E_{L}^{*}, E_{H}^{*}$ are their intrinsic excitation energies. The rotational energy of deformed fragments is due to angular momentum bearing collective modes like bending or wriggling and as such is not drained from the intrinsic excitation energy available at scission [24]. The rotational energy $E^{\text {rot }}$ is simply expressed considering a rotating liquid drop:

$$
E^{\mathrm{rot}}=\frac{\hbar^{2} J(J+1)}{2 \mathcal{I}},
$$

where $J$ stands for the total angular momentum and $\mathcal{I}$ the moment of inertia of nucleus. In the code this moment of inertia is a proportion $k_{\text {rig }}$ of a rigid spheroid $\mathcal{I}_{\text {rig }}$ defined by

$$
\mathcal{I}_{\text {rig }}=\frac{2}{5} A M R^{2}\left(1+0.31 \beta_{2}+0.44 \beta_{2}^{2}+\cdots\right),
$$

where $A, M, R$ and $\beta_{2}$, respectively, stand for the mass number, the nucleon mass, the radius $\left(R=1.2 A^{1 / 3} \mathrm{fm}\right)$ and the quadrupole deformation parameter of the nucleus in its ground state taken from Myers-Swiatecki. Other models have been used, coming from AMEDEE database, for instance [25], and analysis are under progress. The total amount of excitation energy is deduced from the energy release during the fission process $Q$ based on binding energies from references [26] and [27] and the sampled total kinetic energy:

$$
T X E=Q-T K E+E_{n}+B_{n} .
$$

Here $E_{n}$ and $B_{n}$ are the incident neutron energy and the neutron binding energy. Only the intrinsic excitation energy $E^{*}$ corresponding to $T X E-E^{\text {rot }}$ is treated within a Fermi gas approximation in $a T^{2}$, where $a$ and $T$ stand for the level density parameter and the nuclear temperature. An iterative procedure is used to determine the fragment excitation energy because the level density parameter is energy/temperature dependent following the Ignatyuk prescription:

$$
a=\bar{a}\left\{1+\frac{\delta W}{U^{*}}\left(1-e^{-\gamma U^{*}}\right)\right\},
$$

where $\bar{a}(A)$ is the asymptotic level density parameter, $\delta W$ accounts for the shell corrections that can be estimated by the difference between measured and calculated mass excesses (with a liquid drop model, for example), and $U^{*}=E^{*}-\Delta$ is an effective excitation energy function corrected by pairing $\Delta$. The factor $\gamma$ stands for the damping factor. Several implementations of these parameters are driven through RIPL-3 [32]. Even if shell correction and pairing energy are not defined in the same way by various authors (see, for instance, refs. $[28,29]$ or [30]), the whole set of parameters $(\bar{a}, \delta W, \Delta$ and $\gamma)$ involved in eq. (7) must be consistent. For a given fragmentation, the temperature of the light fragment $T_{L}=T\left(A_{L}\right)$ is linked to the temperature of its heavy partner $T_{H}=T\left(A_{H}\right)$ by a massdependent temperature ratio law $R_{T}(A)=T_{L} / T_{H}$ (fig. 4). This law has been already described elsewhere [6]. Briefly the maximum of this law $\left(R_{T}^{\max }\right)$ is supposed to correspond to a minimum temperature of the heavy fragment which is linked to a minimum in the deformation energy (minimum in the excitation energy). This minimum occurs for the doubly closed spherical shell nuclei $(Z=50, N=82$ and $A=132)$. The situation is completely reversed with a minimum in the temperature ratio $\left(R_{T}^{\mathrm{min}}\right)$ when the light fragment has a proton and a neutron closed spherical shell $(Z=28, N=50$ and $A=78)$. Finally for symmetric fission, the temperature of the two identical complementary fragment is the same, the ratio is equal to one. Between these anchorage points, the evolution is supposed to be linear. Once this law is established, all the ingredients are known and can be used to calculate the excitation energies of the two complementary fragments through the iterative scheme:

$$
\left\{\begin{array}{l}
E_{L}^{*}=\frac{T X E-E^{\mathrm{rot}}}{1+\frac{a_{H}}{a_{L} R_{T}^{2}}} \\
E_{H}^{*}=\frac{T X E-E^{\mathrm{rot}}}{1+\frac{a_{L} R_{T}^{2}}{a_{H}}}
\end{array}\right.
$$

At this stage, the characteristics $\left(A, Z, K E, E^{*}, J, \pi\right)$ of the fission fragments are completely known and the deexcitation process can start.

\subsection{De-excitation process: Uncoupled mode}

In the first prompt ejectile evaporation model, neutrons are emitted before gammas (uncoupled scheme). A Weisskopf model is used for neutron emission from initial excitation energy down to a spin-dependent excitation energy 


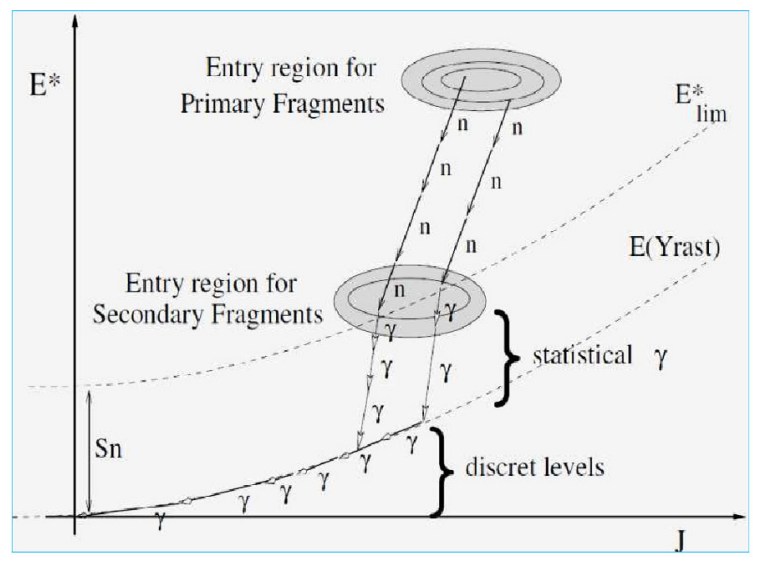

Fig. 5. Sketch of a $n / \gamma$ cascade simulated in FIFRELIN within the uncoupled approach in a $\left[E^{*}, J\right]$ representation.

limit. The centre-of-mass neutron energy $(\epsilon)$ is sampled from the following probability density function:

$$
\phi(\epsilon)=k(T) \epsilon \sigma_{c}(\epsilon) \exp (-\epsilon / T),
$$

where $T$ is the residual temperature, $\sigma_{c}(\epsilon)$ the cross section for the inverse process of compound nucleus formation and $k(T)$ a normalization factor (equal to $T^{-2}$ if the cross section is constant). The residual temperature is recalculated after each emitted neutron. The limit in the neutron evaporation corresponds to the sum of the neutron separation energy $S_{n}$ plus the yrast line of the daughter and is approximated by $E_{\mathrm{lm}}^{*}=S_{n}+E^{\mathrm{rot}}$. Figure 5 is a sketch of an uncoupled $n / \gamma$ cascade in the $\left[E^{*}, J\right]$ plan. Gamma emission is simulated through a Monte Carlo DICEBOXlike approach [31] involving the notion of nuclear realization which is a set of level scheme (energy $E$, spin $J$, parity $\pi$ of a set of levels written $[E J \pi])$ plus the associated partial widths $\Gamma_{p}\left([E J \pi]_{i} \rightarrow[E J \pi]_{f}, \alpha\right)$, where $p$ stands for "particle" and can be a gamma (or a neutron in the coupled mode that will be discussed in the next section. $\alpha$ is related to the quantum numbers ( $X L$ multiplolarity type for gammas, $(l, j)$ orbital and total angular momenta for neutrons). When no experimental data are available (no gamma ray intensities between known levels), only Level Density (LD) and Photon Strength Function (PSF) models are required. These two quantities are directly linked to the gamma transmission. These models are used above an energy cut-off ( $E_{\text {cut-off }}$ that is provided in RIPL-3 [32]) to calculate the partial radiative widths and consequently the set of transition probabilities between a given nuclear state and another one. It can be a group of nuclear states in a given energy bin. Theoretical laws can also be used to complete missing information related to levels (spin for example). Below this energy cut-off energy, the nuclear level scheme, again provided in RIPL-3, is supposed to be complete and the level to level experimental intensities are used to complete the knowledge of the possible cascades down to the ground state or down to an isomeric level depending on the maximum half-life (this value can be fixed by the user). If the nuclear scheme is not completely known (spin information missing for a given level

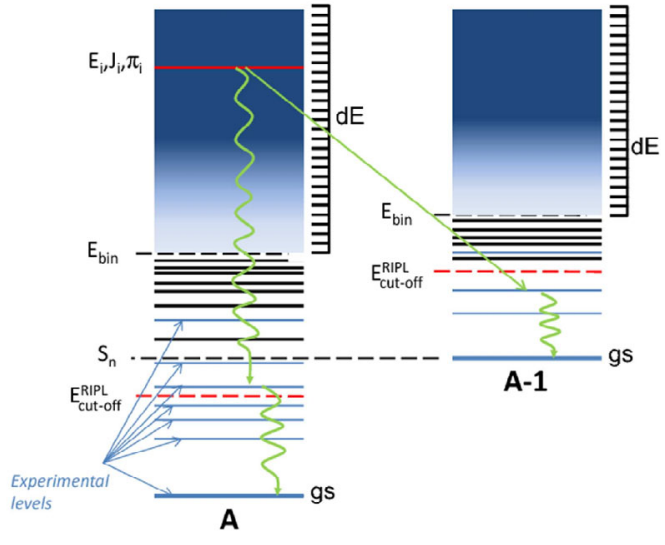

Fig. 6. Sketch of a $n / \gamma$ cascade simulated in FIFRELIN within the $n / \gamma$ coupled approach.

for example) then it is sampled according to a theoretical law. Sometimes, some additional levels have been experimentally observed above $E_{\text {cut-off }}$. In FIFRELIN, these nuclear levels are taken into account up to an upper energy limit $E_{\text {bin }}$ and the level scheme is completed accordingly to a theoretical level density model between $E_{\text {cut-off }}$ and $E_{\text {bin }}$. This upper energy limit is set by a user free parameter corresponding to a maximum level density value (e.g. $10^{5}$ levels $/ \mathrm{MeV}$ ). The algorithm is described in details in a submitted paper [33]. The spin-dependent excitation energy limit for neutron emission allows accounting for neutron/gamma competition simply because gamma emission is favored at high spins compared to neutron emission that rapidly feed up the yrast line. In the next section, the coupled algorithm can account for this competition in a more rigorous way.

\subsection{De-excitation process: Coupled mode}

In a second model the $n / \gamma$ competition is explicitly taken into account. The statistical de-excitation process in an energy, spin, parity ensemble, often called Hauser-Feshbach formalism, has been recently implemented in [13]. Figure 6 shows an example of two possible cascades with or without neutron emission. Gamma emission is treated as previously described in the uncoupled mode (through partial radiation widths) but here neutron emission is taken into account by pre-tabulated neutrontransmission coefficients at the very beginning stage of the de-excitation. It allows describing the transition zone (in energy-spin coordinates) between neutron and gamma emission in a more rigorous way compared to previous uncoupled de-excitation mode because the spin sequence of each fragment is respected during the cascade. On the other hand, it allows simulating a potential gamma emission before the end of the neutron cascade (nevertheless we have observed that a very low amount of gammas is emitted before the last neutron). The neutron transmission coefficients are calculated with the TALYS-1.4 code [34] as a ECIS driver code using Koning-Delaroche or JeukenLejeunne-Mahaux optical model potential. These neutron 
transmission coefficients are tabulated and stored in a FIFRELIN-readable library for 1000 points energy grid from $1 \mathrm{eV}$ to $20 \mathrm{MeV}$, around 600 nuclei and 20 values of the orbital angular momentum.

Briefly, the models available in FIFRELIN for the calculation of level densities are the Constant Temperature Model (CTM), the Composite Gilbert-Cameron Model (CGCM) [28], tabulated values from Hartree-Fock- Bogolyubov model (HFB) provided in a RIPL-3 specific file, or the Generalized Super Fluid Model (GSM) recently implemented by Ducasse [35]. The spin cut-off parameter involved in the calculation of the spin-dependent level density $\rho\left(E^{*}, J, \pi\right)$, is energy dependent as recommended in RIPL-3. It follows the Back shifted Fermi gas model (BSFGM) at high energy, typically $E^{*}>S_{n}\left(\sigma_{F}^{2}\left(E^{*}\right)\right)$ and a discrete value $\left(\sigma_{d}^{2}\right)$ based on the knowledge of the discrete level sequence at low energy. At intermediate energies, the spin cut-off is linearly dependent of the energy.

The implemented closed-form expressions for electric dipole E1 gamma strength functions are the Standard Lorentzian model (SLO) and the Enhanced Generalized Lorentzian model (EGLO). In addition, a tabulated set of values calculated from microscopic approaches such as the Quasi particle Random Phase Approximation (QRPA) model based on SLy4 Skyrme force [36,37]. Ground state is calculated on the same force within the Hartree-Fock + Bardeen-Cooper-Schrieffer model (HF+BCS). Note that these predictions have been improved replacing $\mathrm{HF}+\mathrm{BCS}$ by HFB equations but not directly provided in RIPL-3.

Finally the main models retained in this work for the decay process are based on the Hauser-Feshbach formalism using Composite Gilbert Cameron model for level densities, Enhanced Generalized Lorentzian for electric dipole photon strength functions (other XL-type transitions are calculated within the RIPL-3 prescription) and KoningDelaroche optical model potential for neutron transmission coefficients. These options have been selected among others because they allow to describe in a better agreement most of the fission observables [38].

The five free model parameters that have been described here above $\left(R_{T}^{\min }, R_{T}^{\max }, k_{\text {rig. }}, \overline{\sigma_{L}^{2}}, \overline{\sigma_{H}^{2}}\right)$ are found to reproduce a restricted set of fission observables for each fissioning system (e.g. the average prompt neutron multiplicity $\bar{\nu}$ for ${ }^{239} \mathrm{Pu}(n, f)$, and $\overline{\nu_{L}}, \overline{\nu_{H}}$ for ${ }^{252} \mathrm{Cf}(s f)$ and ${ }^{235} \mathrm{U}(n, f)$. Once these scalar values are achieved with a reasonable agreement, other observables can be discussed.

\section{Total fission fragment kinetic energy}

The total kinetic energy of the fission fragments before neutron emission sampled by the code from experimental data is in good agreement with the recommended values reported by Gönnenwein in [39] as shown in table 1 for ${ }^{252} \mathrm{Cf}(s f),{ }^{235} \mathrm{U}\left(n_{\mathrm{th}}, f\right)$ and ${ }^{239} \mathrm{Pu}\left(n_{\mathrm{th}}, f\right)$ reactions. After neutron emission, the residual total kinetic energy is generally roughly estimated from

$$
\langle T K E\rangle^{\text {post }}=\langle T K E\rangle^{\text {pre }} \frac{A_{C N}-\bar{\nu}}{A_{C N}},
$$

Table 1. Fission fragment total kinetic energies. The statistical uncertainty $(1 \sigma)$ in the Monte Carlo calculations does not exceed $50 \mathrm{keV}$.

\begin{tabular}{lllll}
\hline & \multicolumn{2}{l}{$\langle\text { TKE }\rangle^{\text {pre }}$} & \multicolumn{2}{l}{$\langle\text { TKE }\rangle^{\text {post }}$} \\
& $(\mathrm{MeV})$ & & $(\mathrm{MeV})$ & \\
& Calc. & Ref. [39] & Calc. & Ref. [39] \\
${ }^{252} \mathrm{Cf}(s f)$ & 184.4 & $184.1 \pm 1.3$ & 181.6 & 181.35 \\
${ }^{235} \mathrm{U}\left(n_{\mathrm{th}}, f\right)$ & 170.2 & $170.5 \pm 0.5$ & 168.3 & 168.7 \\
${ }^{239} \mathrm{Pu}\left(n_{\mathrm{th}}, f\right)$ & 177.3 & $177.9 \pm 0.5$ & 175.1 & 175.2 \\
\hline
\end{tabular}

which leads to fission product recommended values that are quite well reproduced from the whole simulation of the $n / \gamma$ cascade (see table 1 ).

\section{Prompt fission neutrons}

In this section we report some results obtained in the case of spontaneous fission of ${ }^{252} \mathrm{Cf}$ and thermal neutroninduced fission of ${ }^{235} \mathrm{U}$. The 5 free model parameters of the simulation were found to reproduce the total average prompt neutron multiplicities of the light and heavy fission fragment groups of [40]

$$
\bar{\nu}_{L}=2.05 \quad \text { and } \quad \bar{\nu}_{H}=1.70,
$$

but we have to keep in mind that the set of five parameters used here is not unique. In the case of thermal fission of ${ }^{235} \mathrm{U}$, the 5 free model parameters were found to reproduce the values from [41]

$$
\bar{\nu}_{L}=1.41 \quad \text { and } \quad \bar{\nu}_{H}=1.01,
$$

but, once again, the five parameters are not a unique set.

\subsection{Multiplicity}

In the case of ${ }^{252} \mathrm{Cf}(s f)$ decay, the calculation of the average prompt fission neutron multiplicity as a function of mass number (so called saw-tooth) is reported in fig. 7 and compared with experimental results from $[40,42,43]$ and [44]. The minimum around $A=132$ obtained in the calculation is driven by two phenomena. First the level density parameter shows a minimum at $A=132$ and second the $R_{T}(A)=T_{L} / T_{H}$ law is maximum at $A=132$ meaning that the temperature of the heavy fragment is minimum. This leads to a minimum in the average number of emitted neutrons. The measured minimum seems to appear at $A=130$ with a mass resolution of about 2 mass units, while the maximum appears for the complementary mass around $A=122$. It is not clear that this minimum always correspond to $A=130$ for other fissioning systems.

In the case of ${ }^{235} \mathrm{U}\left(n_{\mathrm{th}}, f\right)$ reaction, the calculation of the average prompt fission neutron multiplicity as a function of mass number is reported in fig. 8 and compared with experimental results from $[41,45,46]$ and [47]. The 


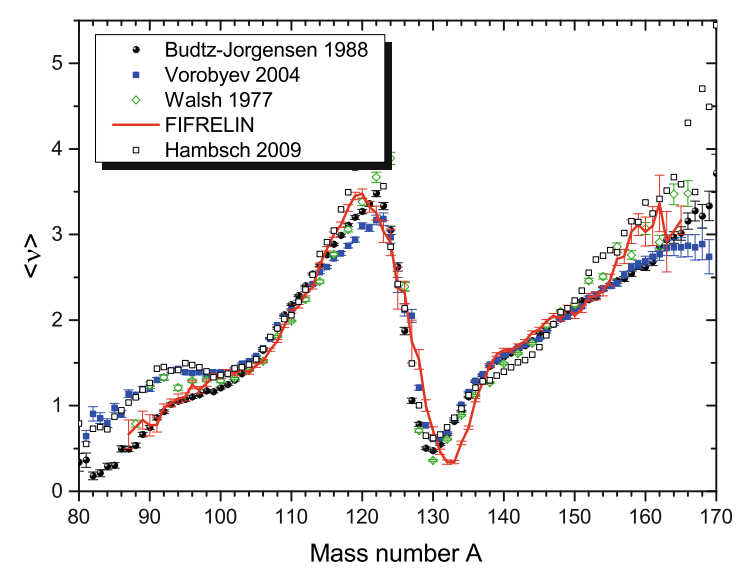

Fig. 7. Average prompt fission neutron multiplicity as a function of fission fragment mass. Comparison between FIFRELIN and experimental data for the ${ }^{252} \mathrm{Cf}(s f)$ spontaneous fission.

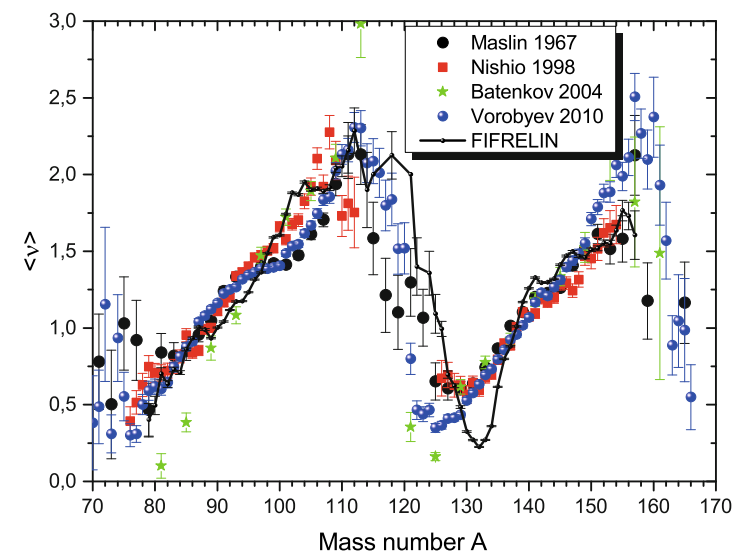

Fig. 8. Average prompt fission neutron multiplicity as a function of fission fragment mass. Comparison between FIFRELIN and experimental data for the ${ }^{235} \mathrm{U}\left(n_{\text {th }}, f\right)$ reaction.

calculation exhibits a minimum around mass 132 as expected by the model discussed above. In experimental data this minimum is lying between mass 126 and mass 132 . We plan to modify the $R_{T}(A)$ law in $\left(e . g .\right.$, a $R_{T}(Z, N)$ law $)$ to study the evolution of this minimum in the saw-tooth shapes.

\subsection{Energy}

The calculation of the average prompt fission neutron energy in the centre of mass as a function of mass number is reported in fig. 9 and compared with experimental results from [42]. The recent results obtained in this work with a new release of the FIFRELIN Monte Carlo code are improved compared with our previous reported results [6].

The prompt fission neutron spectrum (PFNS) calculated in the laboratory frame is shown in fig. 10 for the ${ }^{252} \mathrm{Cf}(s f)$ decay and compared with the reference evaluation from [48]. The PFNS in the case of thermal fission of ${ }^{235} \mathrm{U}$ is reported in fig. 11 for three different models: i) a usual FIFRELIN calculation using CGCM/EGLO/KD options (blue line), ii) the same calculation by replacing

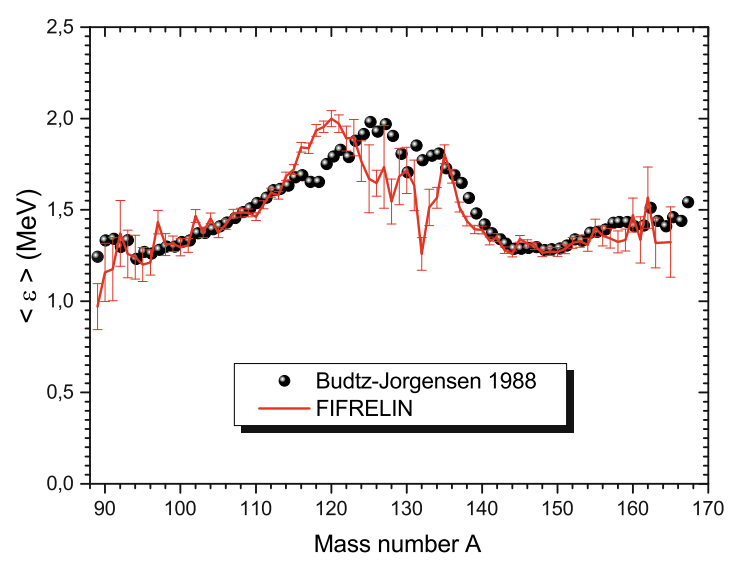

Fig. 9. Average prompt fission neutron energy in the centre of mass frame $\langle\epsilon\rangle$ as a function of fission fragment mass for the ${ }^{252} \mathrm{Cf}(s f)$ decay. Comparison between FIFRELIN model and experimental data [42].

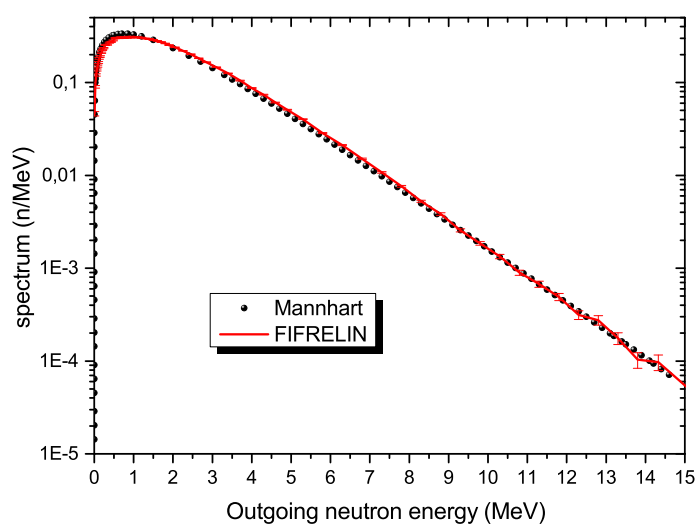

Fig. 10. Prompt fission neutron spectrum for ${ }^{252} \mathrm{Cf}(s f)$. Comparison between FIFRELIN and Mannhart reference evaluation [48].

the CGCM level density model by tabulated values from HFB microscopic calculations (black line) and iii) and an uncoupled-mode calculation using a Weisskopf model for neutrons before gamma emission (red line). These calculations are compared with the JEFF-3.2 evaluation which is based on Madland-Nix PFNS model and several experimental data from [49-51] and [52]. All these spectra are normalized to unity. In the previous sections, we have reported that, especially for $\left.{ }^{252} \mathrm{Cf}(s f), \mathrm{i}\right)$ the total kinetic energy before and after neutron emission were reproduced with a good agreement compared to measurements, ii) the average prompt neutron multiplicity as a function of fragment mass is also well reproduced and finally iii) the mean prompt fission neutron energy in the center of mass as a function of fragment mass seems to be very consistent with measurements (maybe except in the vicinity of masses 130 to 132). But despite these observations, the prompt fission neutron spectrum is still not perfectly reproduced. We may suppose that an anisotropic source of neutrons in the center of mass could help us to reach a better neutron spectrum in the laboratory frame. We have to keep in 


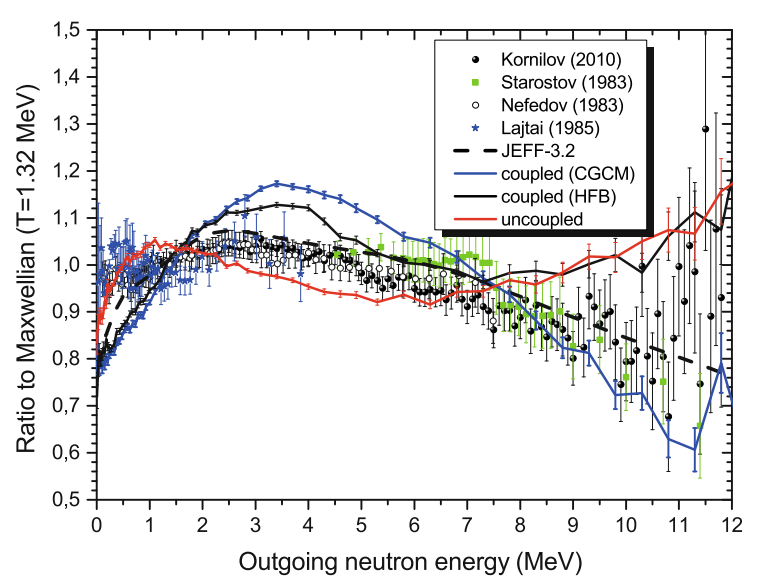

Fig. 11. Ratio of the prompt fission neutron spectrum to a Maxwellian $(T=1.32 \mathrm{MeV})$ for ${ }^{235} \mathrm{U}\left(n_{\mathrm{th}}, f\right)$. Comparison between various FIFRELIN models and experimental data. Blue line is the usual FIFRELIN calculation (CGCM/EGLO/KD), black line corresponds to a change in the level density model (from CGCM to HFB) and red line is an uncoupled mode calculation. The truth is lying in between these models.

mind that we do not simulate any scission neutrons in this work, nor neutron emission during acceleration. If these neutrons exist their spectrum is expected to be different than an evaporation spectrum because they are emitted from a different mechanism. It may explain (at least partly) the differences between calculation and experimental data. Terrell [53] following Hill and Wheeler [54] suggested a preference for neutron emission parallel and antiparallel to fragment velocity in the laboratory frame. They mention that this is probable if fragments are distorted at the moment of emission. This is compatible with an emission during acceleration (or at scission) before fragments have recovered their ground state deformation. In addition Matsumoto et al. [55] following Ohsawa [56] consider that neutron emission in the center of mass frame could be anisotropic due to high angular momenta of fission fragments (also reported by Märten and Ruben from Dresden group [57]) and improve their PFNS calculation at least at low energy. The anisotropic source of neutron emission is still under debate and is used at the time being by different authors as an additional parameter in order to better reproduce experimental data. Last but not least a systematic bias could occur in experimental spectra at low energy if neutron multiple scattering is not well accounted for during the data reduction process [58].

\section{Prompt fission gammas}

\subsection{Multiplicity}

The calculation of the average prompt fission gamma multiplicity as a function of fragment mass number $\bar{M}_{\gamma}(A)$ is reported in figs. 12 and 13 compared with experimental data digitized from [39] (see also ref. [59]) and from [60] respectively. Looking at experimental data, there is no saw-tooth structure for the ${ }^{252} \mathrm{Cf}(s f)$ decay (fig. 12) from

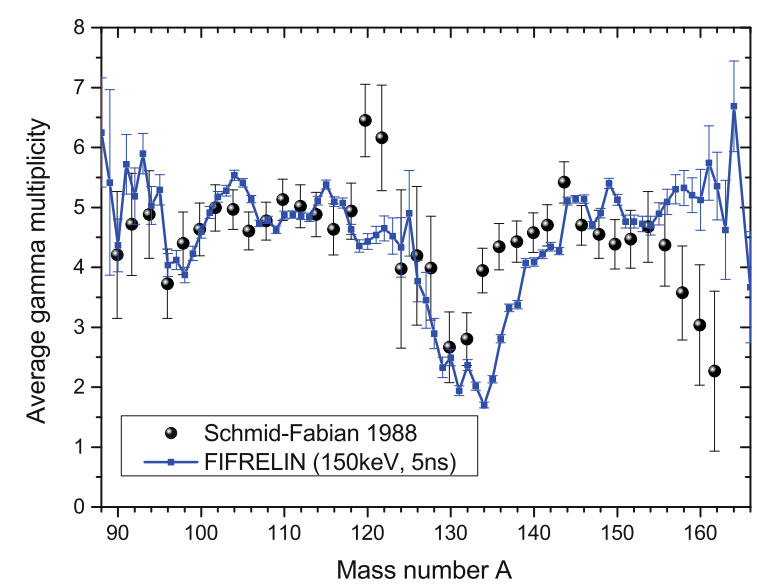

Fig. 12. Average prompt fission gamma multiplicity $\bar{M}_{\gamma}$ as a function of fission fragment mass $A$. Comparison between FIFRELIN model and experimental data digitized from [39] for the ${ }^{252} \mathrm{Cf}(s f)$ decay. Schmid-Fabian 1988 in the legend refers to experimental results reported by Glässel et al. in [59] (threshold is set to $150 \mathrm{keV}$ ).

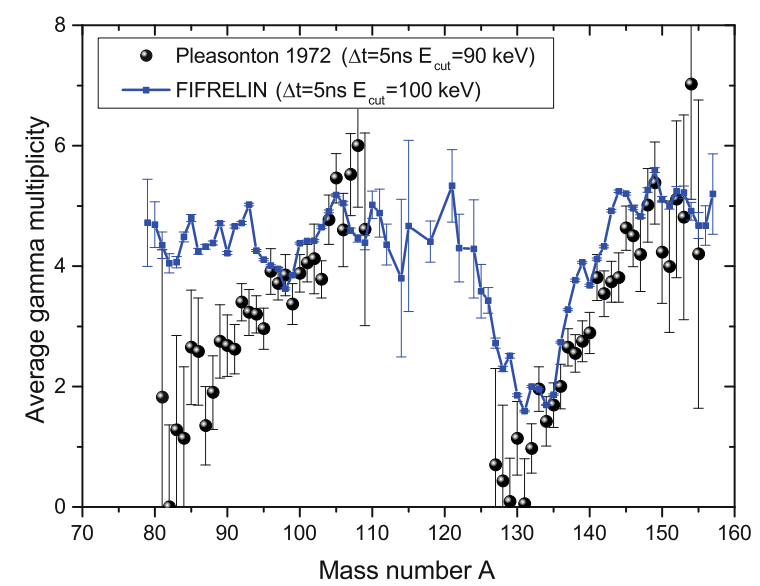

Fig. 13. Average prompt fission gamma multiplicity as a function of fission fragment mass. Comparison between FIFRELIN model and experimental data [60] for the ${ }^{235} \mathrm{U}\left(n_{\mathrm{th}}, f\right)$ reaction.

Glässel and co-workers [59] but a structure appears for the ${ }^{235} \mathrm{U}\left(n_{\mathrm{th}}, f\right)$ reaction (fig. 13$)$.

Our calculations exhibit a rather flat behavior except in the [125-135] mass range where a net lower gammamultiplicity related to near spherical nuclei appears. The same trend has been also observed for the ${ }^{239} \mathrm{Pu}\left(n_{\mathrm{th}}, f\right)$ reaction [61]. Calculations performed with a similar code lead to the same conclusion [62].

We have tested the influence of the initial spin distribution on this fission observable. Here a constant spin cut-off (or $J_{\text {rms }}$ ) is used but if a mass dependence is considered (with a "saw-tooth shape" obtained from two linear relations from light and heavy fragment groups such as $\left.\sigma\left(A_{L, H}\right)=a A_{L, H}+b\right)$ then the average gamma multiplicity exhibits a saw-tooth shape as a function of mass as observed in fig. 14 from a preliminary calculation [63].

The saw-tooth behavior of the average gamma multiplicity as a function of mass is still an open question. 


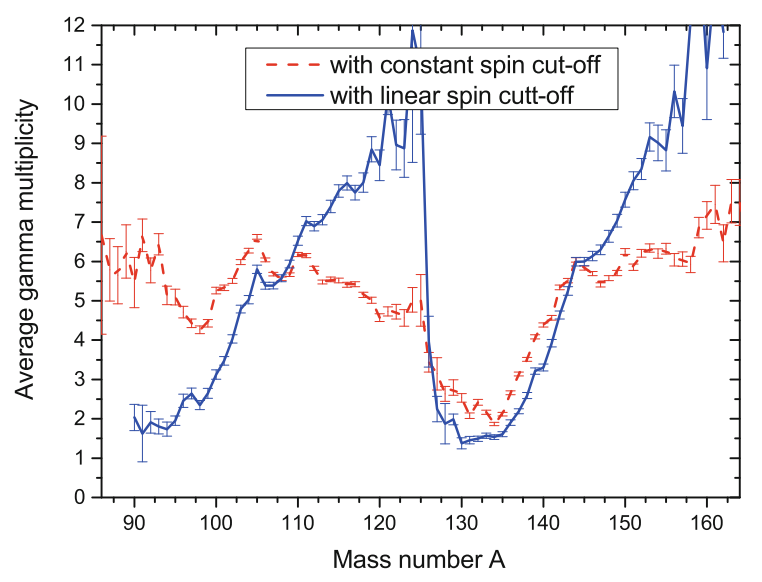

Fig. 14. Influence of the primary spin cut-off parameter on the average gamma multiplicity as a function of mass. The use of a constant spin cut-off parameter for light and heavy fragment groups is compared with a linear dependence as a function of mass (saw-tooth shape). It is clear that a saw-tooth shape in the initial spin cut-off leads to a saw tooth shape in the average gamma multiplicity (blue full line).

Different experiments give different trends and recent experiments (years 1990s) compared to previous ones (years 1960 s to 1970 s) exhibit a flat behavior (see [59] for related discussions). For instance Glässel et al. questioned the collimator method employed in the 1960s that could give wrong information about $\bar{M}_{\gamma}(A)$. As shown in fig. 14 a saw-tooth shape of $J_{\text {rms }}(A)$ allows reproducing a sawtooth shape of $\bar{M}_{\gamma}(A)$. Nevertheless since $J_{\text {rms }}$ is extremely difficult to extract from fission observables, it is still not clear if $J_{\mathrm{rms}}$ presents a saw-tooth or a flat behavior.

\subsubsection{Influence of threshold and maximum half-life}

Two parameters of the simulation must be taken into account when comparing prompt fission gamma related observables. These two parameters are the detection threshold in energy and the coincidence time window (coincidence between fragments and gammas).

The first is obviously easy to handle by simply not recording events below the energy threshold. The higher the threshold, the lower the multiplicity (the higher the mean energy per quantum). This energy threshold is of crucial importance for gammas compared to neutrons because of the gamma multiplicity which is still very high at low energies.

The second parameter can be simulated by taking into account the half life of nuclear levels. If we impose in the simulation a maximum half-life of $10 \mathrm{~ns}$, for instance, only gammas depopulating a level with half life lower than $10 \mathrm{~ns}$ will be simulated (the level is then considered as stable or isomeric, not decaying). If we increase (decrease) this limit, the multiplicity increases (decreases). Figures 15 and 16 show the influence of these parameters on the prompt fission gamma multiplicity distribution $P\left(M_{\gamma}\right)$.

Figure 17 is a simple illustration of the difference in the gamma spectra simulated with $1 \mathrm{~ns}$ and $10 \mathrm{~ns}$ maximum half life. If we focus on the component of the spectrum coming from ${ }^{93} \mathrm{Rb}$, the nuclear level at $266.83 \mathrm{keV}$

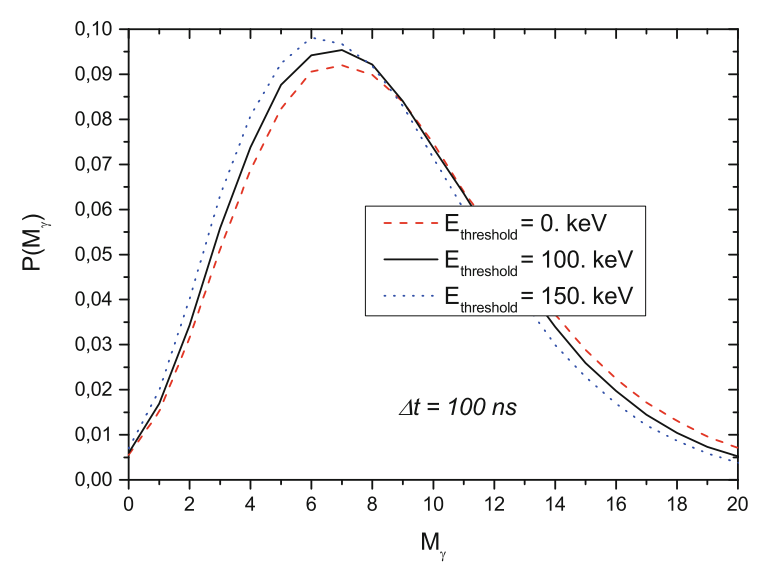

Fig. 15. Influence of the detection threshold on the prompt fission gamma multiplicity distribution $P\left(M_{\gamma}\right)$.

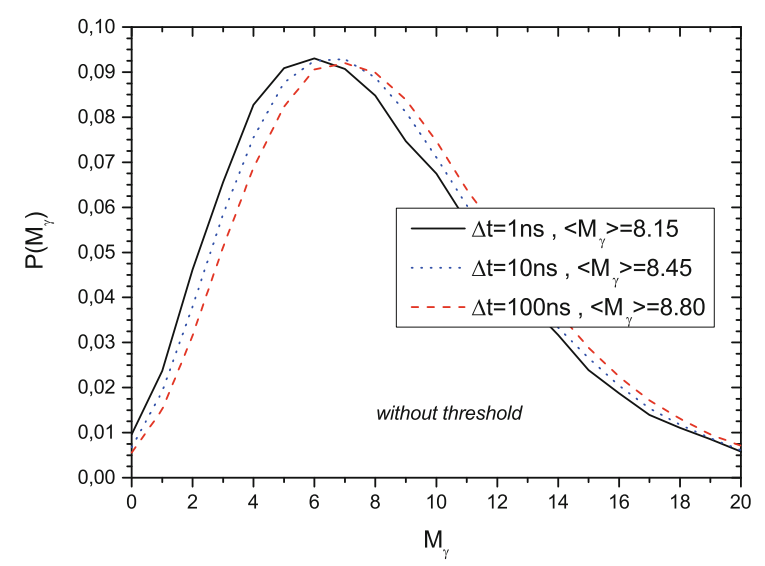

Fig. 16. Influence of the maximum half-life of nuclear levels on the prompt fission gamma multiplicity distribution.

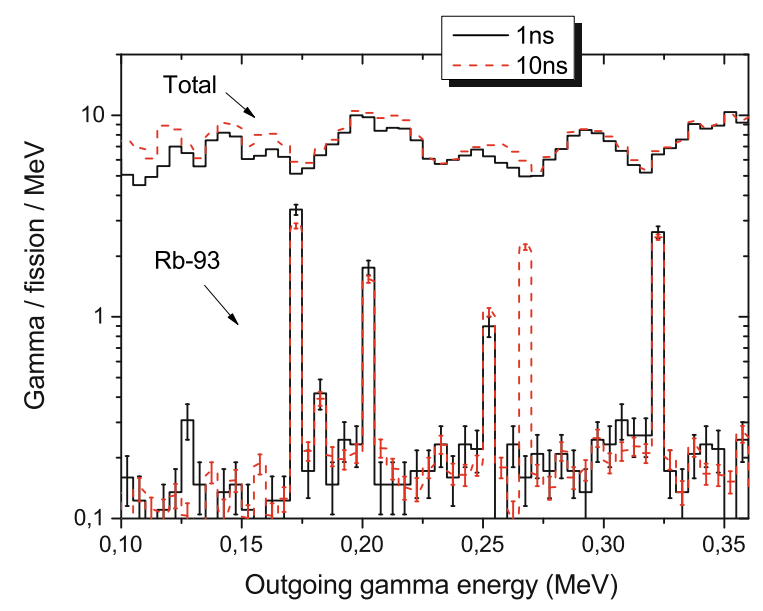

Fig. 17. Low-energy part of the prompt fission gamma spectrum component from ${ }^{93} \mathrm{Rb}$ nucleus for two different maximum half-life of nuclear levels.

with 2.0 ns half life do not de-excites in the first case but de-excites in the second. Consequently the many levels of the numerous nuclei with nuclear levels having a half life 


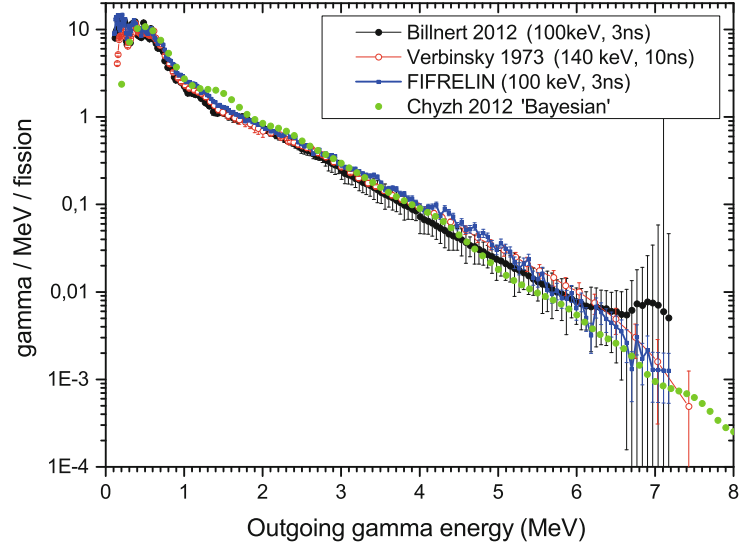

Fig. 18. Prompt fission gamma spectrum for ${ }^{252} \mathrm{Cf}(s f)$. Comparison between FIFRELIN and experimental data from [64, 65] and [66] up to $7 \mathrm{MeV}$ (not rescaled).

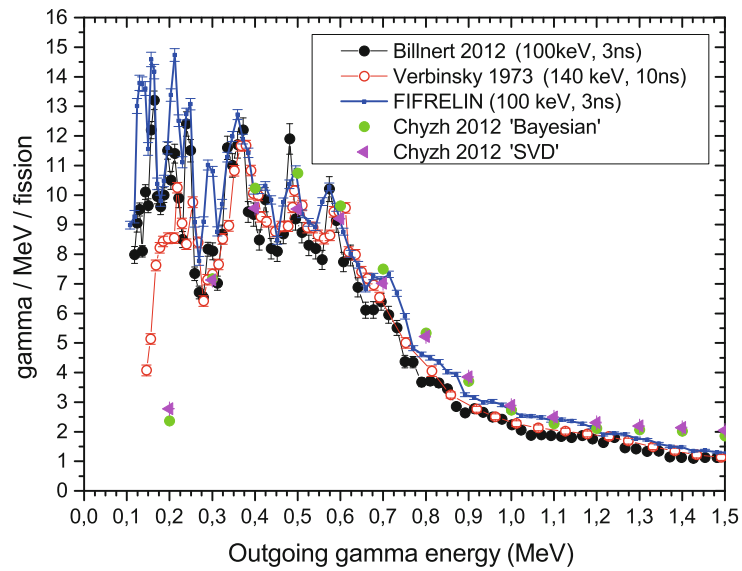

Fig. 19. Prompt fission gamma spectrum for ${ }^{252} \mathrm{Cf}(s f)$. Comparison between FIFRELIN and experimental data from [64, $65]$ and [66] in the low-energy range (not rescaled).

lying in between 1 and $10 \mathrm{~ns}$ will not contribute to the total spectrum in the second case. We would like to stress here that these two parameters (threshold and time window) should be always mentioned when comparing gamma related fission observables.

\subsection{Energy spectra}

Figure 18 shows the prompt fission gamma spectrum in a lin-log representation up to $7 \mathrm{MeV}$ and fig. 19 is a zoom below $1.5 \mathrm{MeV}$ in a lin-lin representation highlighting the overall good agreement between spectra calculated with FIFRELIN and measurements performed by different teams reported in $[64,65]$ or [66]. The structures observed at low energy by Verbinski and co-workers in 1973 have been reproduced in the new measurements by Billnert and co-workers. They found some additional groups of gamma-lines below $200 \mathrm{keV}$ that are also obtained by simulating the whole cascade using, in addition of nuclear models, nuclear structure information associated to lowlying levels. The prompt fission gamma spectrum can be decomposed in its XL-type components such as electric

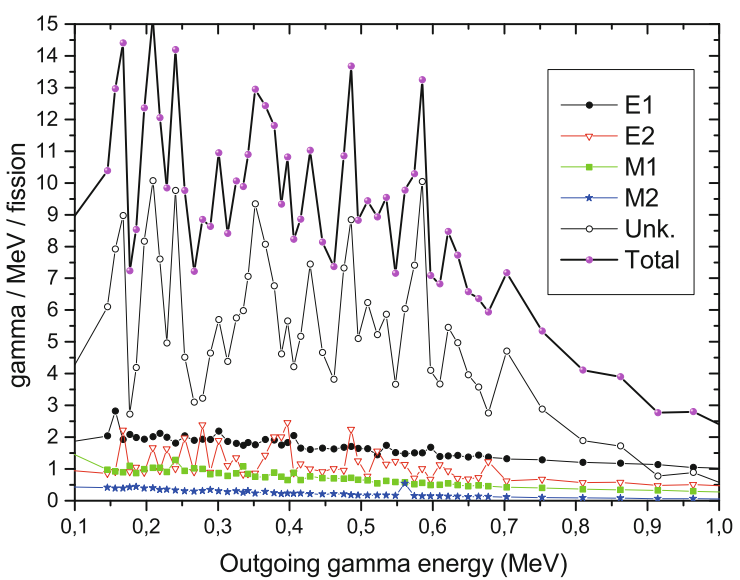

Fig. 20. Decomposition of the ${ }^{252} \mathrm{Cf}(s f)$ calculated PFGS in the centre-of-mass frame relative to XL transition types. "Unk" in the legend stands for transitions with unknown XL type (nevertheless energy and intensity are experimentally determined).

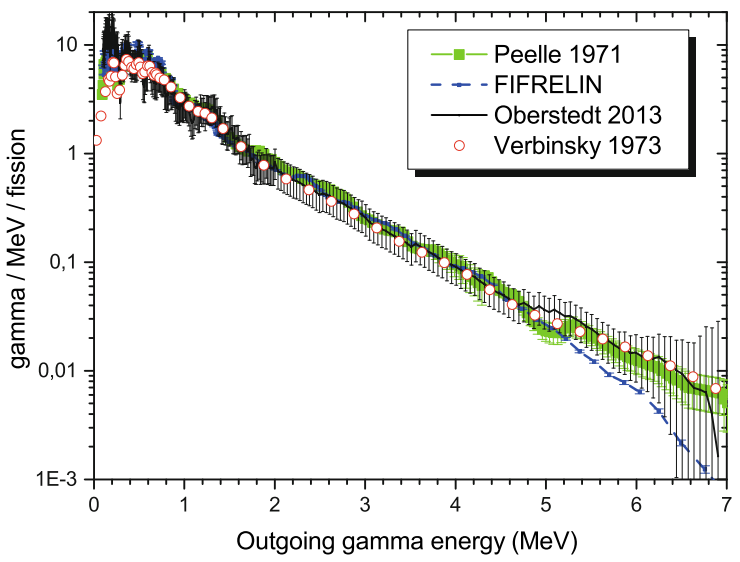

Fig. 21. Prompt fission gamma spectrum for ${ }^{235} \mathrm{U}\left(n_{\mathrm{th}}, f\right)$ reaction up to $7 \mathrm{MeV}$. Comparison between FIFRELIN and experimental data from Verbinski [65], Peelle [67] and Oberstedt [68]. Spectra are not rescaled to the same multiplicity. Threshold and time window used in the calculation are those from [68] $\left(E_{\text {threshold }}=100 \mathrm{keV}, \Delta t=5 \mathrm{~ns}\right)$.

and magnetic dipole, quadrupole and so on, as shown in fig. 20 in the center of mass frame below $1 \mathrm{MeV}$.

As it can be seen, the most part of the total spectrum (full magenta circles connected by black thick line) highlighting separated structures come from experimental transitions corresponding to unknown XL types (open circles connected by black thin line). These transitions appear in the RIPL-3 structure database between known levels (at least in energy) but even if the intensity has been measured, the XL type stay undefined. The other XL known transitions (coming from cascade model in the continuum and from nuclear structure database in the discrete levels scheme) are much lower as expected.

Figures 21 and 22 show the prompt fission gamma spectrum in a lin-log representation up to $7 \mathrm{MeV}$ and below $1.8 \mathrm{MeV}$, respectively. The structures at low energy are observed by Oberstedt and co-workers [68] and in a 


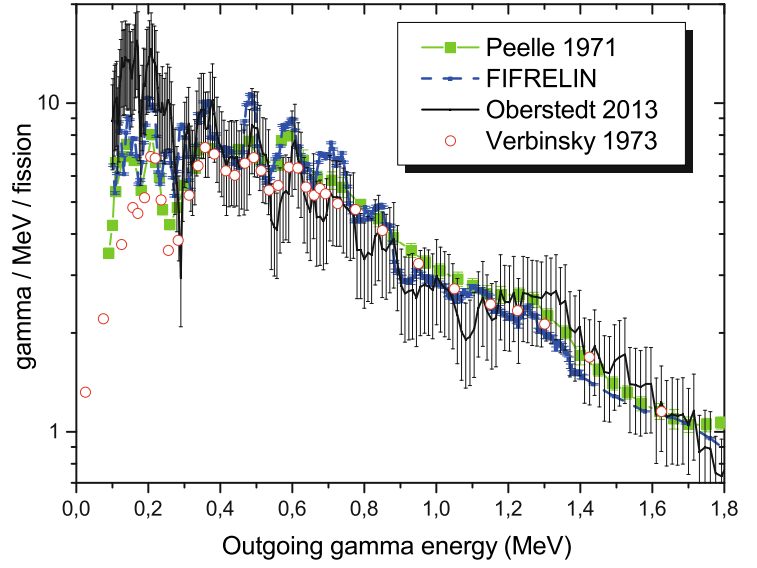

Fig. 22. Prompt fission gamma spectrum for ${ }^{235} \mathrm{U}\left(n_{\mathrm{th}}, f\right)$ reaction below $1.8 \mathrm{MeV}$. Comparison between FIFRELIN and experimental data from Verbinski [65], Peelle [67] and Oberstedt [68]. Spectra are not rescaled to the same multiplicity. Threshold and time window used in the calculation are those from [68] $\left(E_{\text {threshold }}=100 \mathrm{keV}, \Delta t=5 \mathrm{~ns}\right)$.

Table 2. Prompt fission $\gamma$-ray characteristics for ${ }^{235} \mathrm{U}\left(n_{\mathrm{th}}, f\right)$ reaction $\left(E_{\text {threshold }}=100 \mathrm{keV}, \Delta t=5 \mathrm{~ns}\right)$.

\begin{tabular}{llll}
\hline & $\begin{array}{l}\overline{M_{\gamma}} \\
(\gamma / \text { fission })\end{array}$ & $\begin{array}{l}\left\langle\epsilon_{\gamma}\right\rangle \\
(\mathrm{MeV} / \gamma)\end{array}$ & $\begin{array}{l}\left\langle E_{\gamma}^{\text {tot }}\right\rangle \\
(\mathrm{MeV} / \text { fission })\end{array}$ \\
\hline$[68]$ & $8.19 \pm 0.11$ & $0.85 \pm 0.02$ & $6.92 \pm 0.09$ \\
This work & $8.09 \pm 0.01$ & $0.875 \pm 0.001$ & $7.07 \pm 0.01$ \\
\hline
\end{tabular}

lesser extend by Peelle [67] and Verbinski [65] are globally reproduced by the calculation, as in the previous fissioning system.

Table 2 summarizes the prompt average gamma values obtained with the code and compared with results from [68] using the same gamma energy threshold and time window $\left(E_{\text {threshold }}=100 \mathrm{keV}, \Delta t=5 \mathrm{~ns}\right)$.

\section{Beyond common observables}

In this section we would like to stress that because a Monte Carlo simulation of the whole simulation of the neutron/gamma/electron emission by fragments bring a huge amount of quantities, it is possible to check in details the sources of differences between models. To illustrate this fact, we present here the prompt fission gamma spectrum obtained in the case of the thermal fission of ${ }^{235} \mathrm{U}$ with two models related to the gamma de-excitation for the description of level densities and photon strength functions: i) CGCM/EGLO and ii) HFB/QRPA. The following figures show how we can distinguish in more and more details the sources of discrepancies. First we can observe in fig. 23 a higher multiplicity in the low energy range $(E<0.4 \mathrm{MeV})$ spectrum when we use microscopic ingredients (HFB/QRPA) compared to closedform expressions for level density and photon strength functions (CGCM/EGLO). If we look at figs. 24 and 25 showing the average gamma multiplicity as a function of

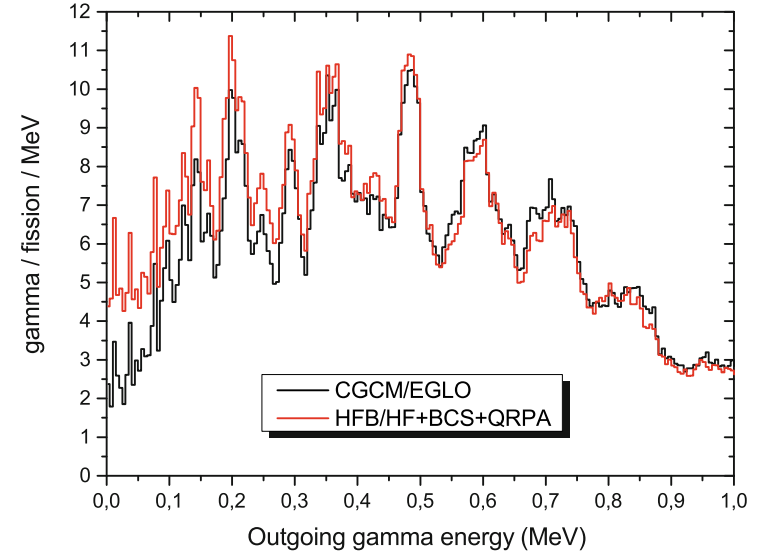

Fig. 23. Prompt fission gamma spectrum obtained with two different level density and strength function models: closedform expressions and microscopic calculations.

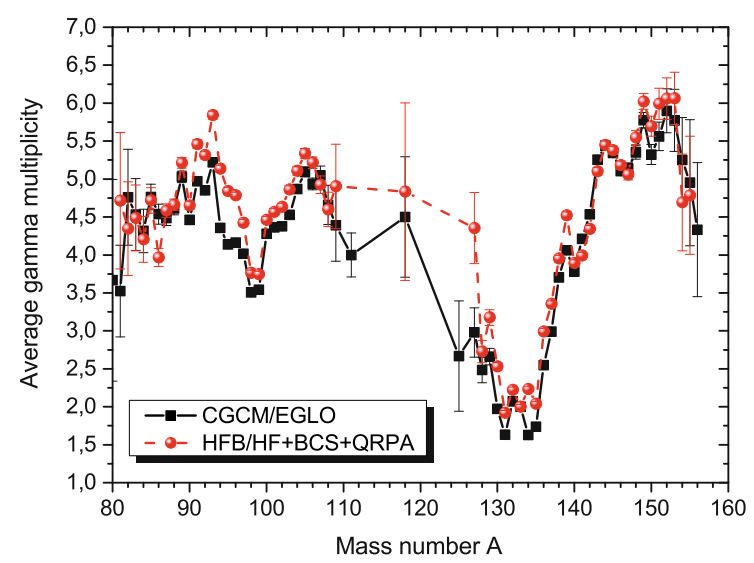

Fig. 24. Average prompt gamma multiplicity as a function of pre-neutron fragment mass for two level densities/strength function models.

fragment mass and charge respectively, we can suppose that Krypton, Rubidium or Strontium isotopes can be responsible of this difference with probably a mass ranging from $A=90$ to $A=110$. As an illustration, the prompt gamma fission spectrum from ${ }^{93} \mathrm{Rb}$ and ${ }^{95} \mathrm{Rb}$ are shown in figs. 26 and 27, respectively. It is then clear that the difference comes from ${ }^{93} \mathrm{Rb}$ with a higher component obtained with microscopic ingredients. An energy spectrum is a collection of radiative transitions between nuclear levels and the calculation of a transition requires the knowledge a level density model and a photon strength function model. If we go further, we can compare the photon strength functions (QRPA and EGLO) for these nuclei (figs. 28 and 29). The parameters used in the EGLO model come from systematic provided in RIPL-3. The two other models are QRPA tabulated values from $\mathrm{HFB}+\mathrm{QRPA}$ and from $\mathrm{HF}+\mathrm{BCS}+\mathrm{QRPA}$. The calculation uses the tabulated values from $\mathrm{HF}+\mathrm{BCS}+\mathrm{QRPA}$ and we can see that the difference between this calculation and a EGLO calculation type is quite the same for ${ }^{93} \mathrm{Rb}$ and ${ }^{95} \mathrm{Rb}$. 


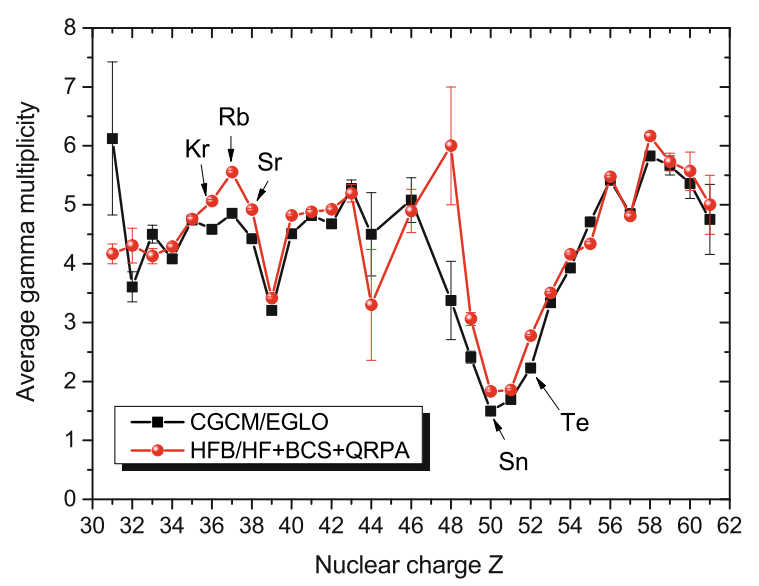

Fig. 25. Average prompt gamma multiplicity as a function of fragment nuclear charge for two level densities/strength function models.

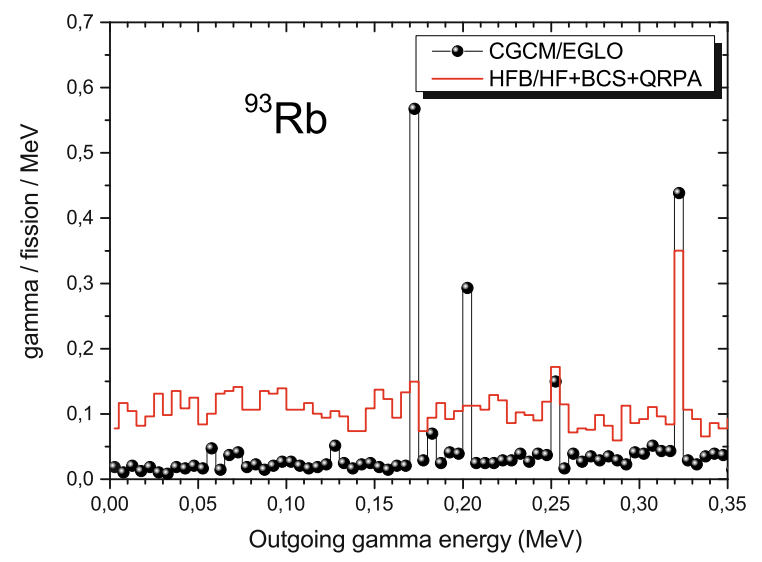

Fig. 26. Prompt fission gamma spectrum from ${ }^{93} \mathrm{Rb}$ obtained with two level density/strength function models.

Consequently, it is the level density model used for ${ }^{93} \mathrm{Rb}$ which is responsible of a number of transitions roughly 5 times higher in HFB compared with CGCM.

\section{Conclusion and perspectives for the next decade}

The models used in the FIFRELIN Monte Carlo code has been described. It allows estimating the whole set of fission observables (prompt component) from particle multiplicity to energy spectra as well as post-neutron kinetic energies, yields and so on. Some results compared with experimental data have been shown highlighting the overall good agreement obtained by comparing calculations and measurements.

Nevertheless, there is room for improvements and the next decade will be certainly intense in brainstorming activities. Different steps in the calculation that can be improved in the next decade are reported in table 3. A Rootbased tree of events [69] could be generated by the code in order to simplify the analysis in a multidimensional parameter space. This kind of job has already been tested in previous studies [23] and should have a regain of interest.

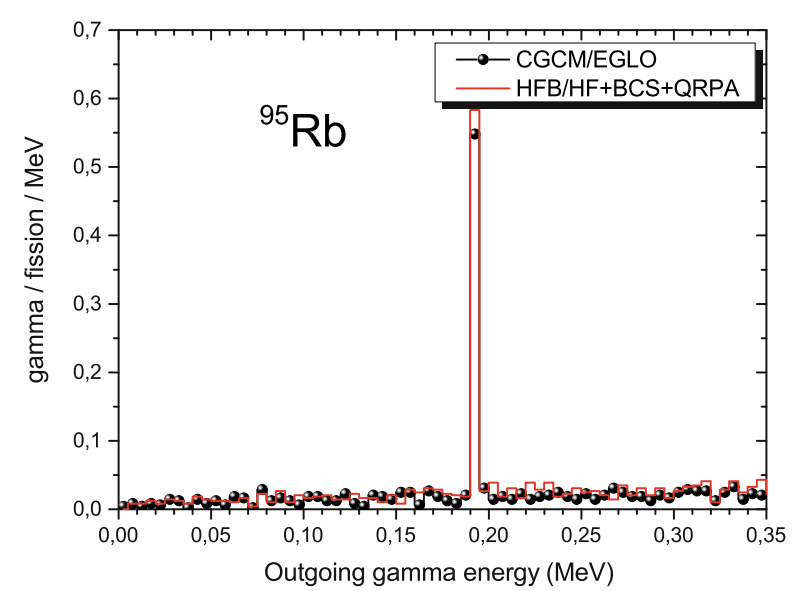

Fig. 27. Prompt fission gamma spectrum from ${ }^{95} \mathrm{Rb}$ obtained with two level density/strength function models.

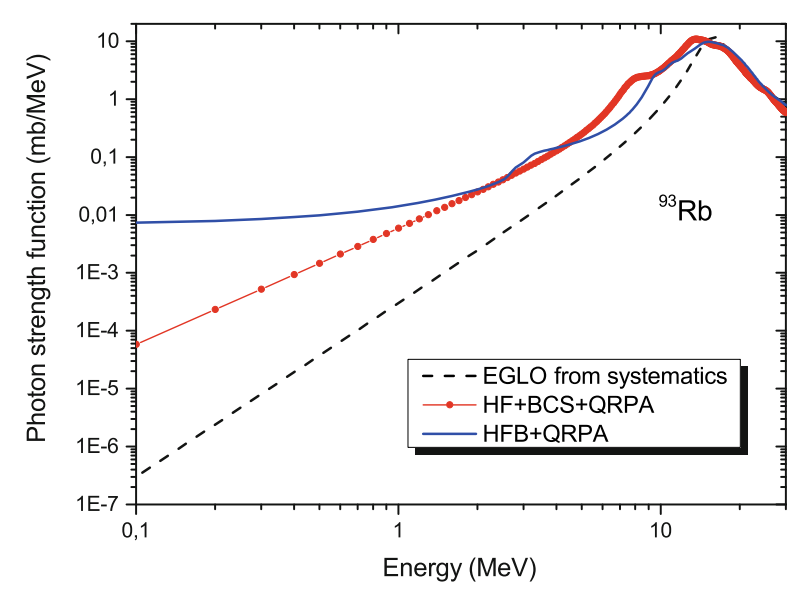

Fig. 28. Photon strength function for ${ }^{93} \mathrm{Rb}$.

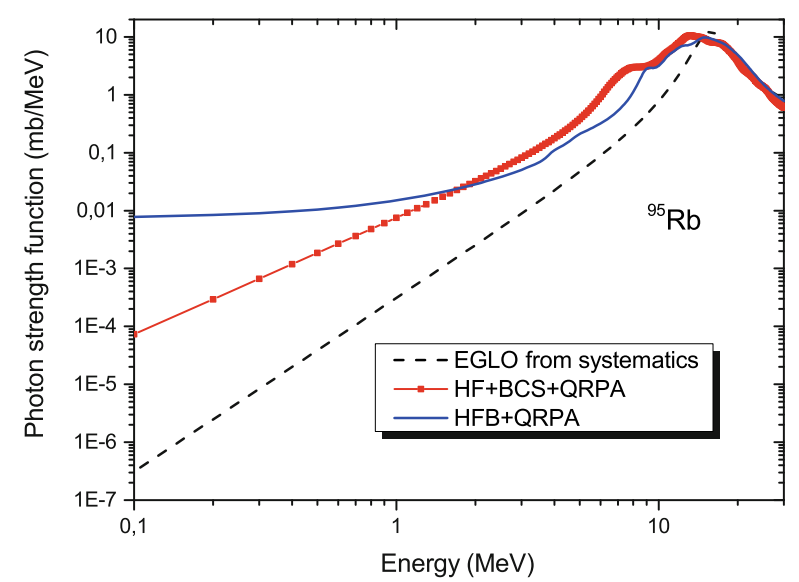

Fig. 29. Photon strength function for ${ }^{95} \mathrm{Rb}$. 
Table 3. Perspectives for fission observable modeling.

\begin{tabular}{|c|c|c|}
\hline Input/Output & Today & Next decade \\
\hline $\begin{array}{l}\text { Mass and kinetic } \\
\text { energy distributions }\end{array}$ & $\begin{array}{l}\text { - Reconstructed from post-neutron data. } \\
\text { - Calculated from fission mode parameters. }\end{array}$ & $\begin{array}{l}\text { - Mass and Kinetic energy matrices provided from } \\
\text { multidimensional Langevin equations }[78,79] \text {. } \\
\text { - Provided by external specific codes. } \\
\text { - Provided from new experimental facilities } \\
\text { (FALSTAFF [70], FIPPS [80], SOFIA }[71], \ldots \text { ). }\end{array}$ \\
\hline Charge distributions & $\begin{array}{l}\text { - Modified Gaussian using Unchanged Charge } \\
\text { Density (UCD) assumption, charge polarization } \\
\text { function and even-odd factors. }\end{array}$ & $\begin{array}{l}\text { - Provided from new experimental facilities } \\
(\text { FALSTAFF, FIPPS, SOFIA,...). }\end{array}$ \\
\hline Spin distributions & $\begin{array}{l}\text { - Spin cut-off formula } \\
\text { (mass and deformation dependent). }\end{array}$ & $\begin{array}{l}\text { - Spin cut-off formula (energy dependent). } \\
\text { - Provided thanks to isomeric ratio } \\
\text { (GFM [81], EXILL [82], FIPPS) } \\
\text { or yields of specific fragment pairs (EXILL). }\end{array}$ \\
\hline $\begin{array}{l}\text { Excitation energy } \\
\text { (sharing between } \\
\text { fragments) }\end{array}$ & $\begin{array}{l}\text { Temperature ratio law } \\
\text { (mass, fission mode dependent). }\end{array}$ & $\begin{array}{l}\text { - From charge-dependent temperature ratio law. } \\
\text { - From microscopic calculations. } \\
\text { - Provided by external codes. }\end{array}$ \\
\hline De-excitation process & $\begin{array}{l}\text { - Level Density model: CGCM/CTM/HFB. } \\
\text { - Photon Strength Function: EGLO/SLO/HFB. } \\
\text { - Optical Model Potential: KD/JLM. }\end{array}$ & $\begin{array}{l}\text { - Other closed-form models. } \\
\text { - HFB+QRPA tabulations. } \\
\text { - Deformed OMP for tabulated neutron } \\
\text { transmission coefficients. }\end{array}$ \\
\hline Other neutron source & $\begin{array}{l}\text { - Neutrons evaporated from fully } \\
\text { accelerated fission fragments. }\end{array}$ & $\begin{array}{l}\text { - Accounting for scission neutrons or } \\
\text { neutron emission during FF acceleration. }\end{array}$ \\
\hline
\end{tabular}

Several experimental setups reported in table 3 (non exhaustive list) will provide numerous fission observables for various fissioning systems in the next decade. Pre-neutron mass yields will be specially appreciated and future experimental setups will certainly contribute [70,71]. A huge effort for measuring prompt fission gammas and neutrons has been already made by colleagues from IRMM (Institute for Reference Materials and Measurements) at Geel, Belgium. New data are already published [72] and will be used to improve our future simulations. Additional valuable data are now available and the activity around this topic is still relevant $[73,74]$ and will continue to be a challenge in the near future [75-77].

A lack of knowledge concerns the spin distribution of fission fragments at least after neutron evaporation and all the efforts from the whole community of experimentalists and theoreticians will be necessary to overcome this crucial point in the characterization of fission observables. Several solutions will be envisaged by using isomeric ratio measurements and still have to be continued [81].
The last comment concerns an activity under progress which is the "simulation of analog fission" in transport codes that requires a coupling with dedicated code simulating fission observables. In this procedure the neutron is no more sampled from average distributions but directly generated from specific codes, fission event by fission event. The uncoupled scheme described at beginning of this paper could be used to fit these objectives but the complete Monte Carlo simulation of the neutron/gamma cascade within a Hauser-Feshbach formalism employed in the coupled scheme is probably unrealistic at the time being. Finally there is still a lot of work to be done in order to understand and describe fission observables.

\section{References}

1. V.F. Weisskopf, Phys. Rev. 52, 295 (1937).

2. S. Lemaire, P. Talou, T. Kawano, M.B. Chadwick, D.G. Madland, Phys. Rev. C 72, 024601 (2005). 
3. S. Lemaire, P. Talou, T. Kawano, M.B. Chadwick, D.G. Madland, Phys. Rev. C 73, 014602 (2006).

4. J. Randrup, R. Vogt, Phys. Rev. C 80, 024601 (2009).

5. K.-H. Schmidt, B. Jurado, Phys. Rev. Lett. 104, 212501 (2010).

6. O. Litaize, O. Serot, Phys. Rev. C 82, 054616 (2010).

7. K.-H. Schmidt, B. Jurado, Phys. Rev. C 83, 061601 (2011).

8. P. Talou, B. Becker, T. Kawano, M.B. Chadwick, Y. Danon, Phys. Rev. C 83, 064612 (2011).

9. R. Vogt, J. Randrup, D.A. Brown, M.A. Descalle, W.E. Ormand, Phys. Rev. C 85, 024608 (2012).

10. D. Regnier, O. Litaize, O. Serot, Phys. Proc. 31, 59 (2012).

11. W. Hauser, H. Feshbach, Phys. Rev. 87, 366 (1952).

12. B. Becker, P. Talou, T. Kawano, Y. Danon, I. Stetcu, Phys. Rev. C 87, 014617 (2013).

13. D. Regnier, O. Litaize, O. Serot, Phys. Proc. 47, 47 (2013).

14. U. Brosa, S. Grossmann, A. Muller, Phys. Rep. 197, 167 (1990).

15. N. Varapai, F.-J. Hambsch, S. Oberstedt, O. Serot, G. Barreau, N. Kornilov, S. Zeinalov, in Proceedings of the International Workshop on Nuclear Fission and Fission Product Spectroscopy, edited by G. Fioni et al., Vol. 447 (Cadarache, France, 2005) p. 369.

16. F.-J. Hambsch, H.-H. Knitter, C. Budtz-Jorgensen, J.-P. Theobald, Nucl. Phys. A 491, 56 (1989).

17. C. Wagemans, E. Allaert, A. Deruytter, R. Barthélémy, P. Schillebeeckx, Phys. Rev. C 30, 218 (1984).

18. L. Demattè, PhD Thesis, University of Gent, Belgium (1997).

19. A.C. Wahl, At. Data Nucl. Data Tables 39, 1 (1988).

20. J.P. Bocquet, R. Brissot, Nucl. Phys. A 502, 213c (1989).

21. H. Naik, S.P. Dange, R.J. Singh, S.B. Manohar, Nucl. Phys. A 612, 143 (1997).

22. H. Naik, R.J. Singh, R.H. Iyer, J. Phys. G: Nucl. Part. Phys. 30, 107 (2004).

23. O. Litaize, O. Serot, D. Regnier, S. Theveny, S. Onde, Phys. Proc. 31, 51 (2012).

24. F. Gönnenwein et al., in Proceedings of Seminar on Fission, edited by C. Wagemans et al., Vol. 3 (Corsendonk Priory, Belgium, 2007).

25. S. Hilaire, M. Girod, Eur. Phys. J. A 33, 237 (2007).

26. A.H. Wapstra, G. Audi, C. Thibault, Nucl. Phys. A $\mathbf{7 2 9}$, 129 (2003).

27. G. Audi, A.H. Wapstra, C. Thibault, Nucl. Phys. A 729, 337 (2003).

28. A. Gilbert, A.G.W. Cameron, Can. J. Phys. 43, 1446 (1965).

29. W.D. Myers, W.J. Swiatecki, Nucl. Phys. A 601, 141 (1996).

30. P. Moller, J.R. Nix, W.D. Myers, W.J. Swiatecki, At. Data Nucl. Data Tables 59, 185 (1995).

31. F. Becvar, Nucl. Instrum. Methods Phys. Res. A 417, 434 (1998).

32. R. Capote et al., Nucl. Data Sheets 110, 3107 (2009).

33. D. Regnier, O. Litaize, O. Serot, to be published in Comput. Phys. Commun.

34. A.J. Koning et al., Proceedings of the International Conference on Nuclear Data for Science and Technology ND200\%, edited by O. Bersillon (Nice, France, 2007) p. 211.

35. Q. Ducasse, private communication.

36. E. Khan et al., Nucl. Phys. A 694, 103 (2001).
37. S. Goriely, E. Khan, Nucl. Phys. A 706, 217 (2002).

38. D. Regnier, PhD Thesis, University of Grenoble, France (2013).

39. C. Wagemans, The Nuclear Fission Process (CRC Press, 1991) p. 480.

40. A.S. Vorobyev, V.N. Dushin, F.-J. Hambsch, V.A. Jakolev, V.A. Kalinin, A.B. Laptev, B.F. Petrov, O.A. Shcherbakov, in Proceedings of the International Conference on $\mathrm{Nu}$ clear Data for Science and Technology ND2004, edited by R.C. Haight et al. (Santa Fe, USA, 2004) p. 613.

41. K. Nishio, Y. Nakagome, H. Yamamoto, I. Kimura, Nucl. Phys. A 632, 540 (1998).

42. C. Budtz-Jørgensen, H.H. Knitter, Nucl. Phys. A 490, 307 (1988).

43. R.L. Walsh, J.W. Boldeman, Nucl. Phys. A 276, 189 (1977).

44. F.-J. Hambsch, private communication.

45. E.E. Maslin, A.L. Rodgers, W.G.F. Core, Phys. Rev. 164, 1920 (1967).

46. O.A. Batenkov et al., AIP Conf. Proc. 769, 1003 (2005).

47. A. Vorobyev, O. Shcherbakov, A. Gagarski, G. Valaski, G. Petrov, EPJ Web of Conferences 8, 03004 (2010).

48. W. Mannhart, in Properties of Neutron Sources, Report IAEA-TECDOC-410 (1987) p. 158.

49. N. Kornilov, F.-J. Hambsch, I. Fabry, S. Oberstedt, T. Belgya, Z. Kis, L. Szentmiklosi, S. Simakov, Nucl. Sci. Eng. 165, 117 (2010).

50. B.I. Starostov, V.N. Nefedov, A.A. Boytzov, Proccedings of the All Union Conference on Neutron Physics, Vol. 2 (Kiev, USSR, 1983) p. 290.

51. V.N. Nefedov, B.I. Starostov, A.A. Boytzov, Proccedings of the All Union Conference on Neutron Physics, Vol. 2 (Kiev, USSR, 1983) p. 285.

52. A. Lajtai, J. Kecskemeti, J. Safar, P.P. Dyachenko, V.M. Piksaikin, Proccedings of the Conference on Nuclear Data for Basic and Applied Sciences, Vol. 1 (Santa Fe, USA, 1985) p. 613.

53. J. Terrell, Phys. Rev. 113, 527 (1959).

54. D.L. Hill, J.A. Wheeler, Phys. Rev. 89, 1102 (1953).

55. A. Mastsumoto, H. Taninaka, K. Hashimoto, T. Ohsawa, J. Nucl. Sci. Technol. 49, 782 (2012).

56. T. Ohsawa, in IAEA Report INDC(NDS)-0541 (2009) p. 71.

57. H. Märten, A. Ruben, Sov. At. Ener. 69, 583 (1990).

58. T.N. Taddeucci et al., Nucl. Data Sheets 123, 135 (2015).

59. P. Glässel, R. Schmid-Fabian, D. Schwalm, D. Habs, H.U.V. Helmolt, Nucl. Phys. A 502, 315c (1989).

60. F. Pleasonton, R.L. Ferguson, H.W. Schmitt, Phys. Rev. C 6, 1023 (1972).

61. O. Serot, O. Litaize, D. Regnier, Phys. Proc. 59, 132 (2014).

62. I. Stetcu, P. Talou, T. Kawano, M. Jandel, Phys. Rev. C 90, 024617 (2014).

63. L. Thulliez, private communication (2015).

64. R. Billnert, F.-J. Hambsch, A. Oberstedt, S. Oberstedt, Phys. Rev. C 87, 024601 (2013).

65. V.V. Verbinski, H. Weber, R.E. Sund, Phys. Rev. C 7, 1173 (1973).

66. A. Chyzh, C.Y. Wu, E. Kwan, R.A. Henderson, J.M. Gostic, T.A. Bredeweg, R.C. Haight, A.C. Hayes-Sterbenz, M. Jandel, J.M. O’ Donnell, J.L. Ullmann, Phys. Rev. C 85, 0216011 (2012). 
67. R.W. Peelle, F.C. Maienschein, Phys. Rev. C 3, 373 (1971).

68. A. Oberstedt, T. Belgya, R. Billnert, R. Borcea, T. Brys, W. Geerts, A. Gook, F.-J. Hambsch, Z. Kis, T. Martinez, S. Oberstedt, L. Szentmiklosi, K. Takacs, M. Vidali, Phys. Rev. C 87, 051602 (2013).

69. R. Brun, F. Rademakers, Nucl. Instrum. Methods Phys. Res. A 389, 81 (1997).

70. D. Doré, F. Farget, F.-R. Lecolley, G. Lehaut, T. Materna, J. Pancin, S. Panebianco, Th. Papaevangelou, EPJ Web of Conferences 62, 05005 (2013).

71. J. Taieb et al., Int. J. Mod. Phys. E 18, 767 (2009).

72. A. Gook, F.-J. Hambsch, M. Vidali, Phys. Rev. C 90 , 064611 (2014)

73. R. Billnert, A. Oberstedt, S. Oberstedt, Phys. Proc. 59, 17 (2014).

74. A. Oberstedt, R. Billnert, S. Oberstedt, Phys. Proc. 59, 24 (2014)
75. J.N. Wilson, M. Leblois, P. Halipre, S. Oberstedt, A. Oberstedt, Phys. Proc. 59, 31 (2014).

76. M. Leblois, J.N. Wilson, P. Halipre, B. Leniau, I. Matea, A. Oberstedt, S. Oberstedt, D. Verney, Phys. Proc. 59, 37 (2014).

77. M. Jandel et al., Phys. Proc. 59, 101 (2014).

78. Y. Aritomo, Proceedings of Nuclear Fission and Structure of Exotic Nuclei - ASRC (Tokai, Japan, 2014).

79. A.J. Sierk, LANL Report LA-UR-14-27056 (2014).

80. A. Blanc et al., in Proceedings of Symposium on Capture Gamma-Ray Spectroscopy and Related Topics CGC15 (Dresden, Germany, 2014).

81. G. Kessedjian, A. Chebboubi, H. Faust, U. Köster, T. Materna, C. Sage, O. Serot, EPJ Web of Conferences 42, 01007 (2013).

82. A. Blanc et al., Nucl. Instrum. Methods Phys. Res. B 317, 333 (2013). 